\title{
How do validated measures of functional outcome compare with commonly used outcomes in administrative database research for lumbar spinal surgery?
}

\author{
Daniel Omoto $\cdot$ S. Samuel Bederman $\cdot$ \\ Albert J. M. Yee $\cdot$ Hans J. Kreder • \\ Joel A. Finkelstein
}

Received: 24 December 2008/Revised: 24 September 2009/Accepted: 24 September 2009/Published online: 9 October 2009

(C) The Author(s) 2009. This article is published with open access at Springerlink.com

\begin{abstract}
Clinical interpretation of health services research based on administrative databases is limited by the lack of patient-reported functional outcome measures. Reoperation, as a surrogate measure for poor outcome, may be biased by preferences of patients and surgeons and may even be planned a priori. Other available administrative data outcomes, such as postoperative cross sectional imaging (PCSI), may better reflect changes in functional outcome. The purpose was to determine if postoperative events captured from administrative databases, namely reoperation and PCSI, reflect outcomes as derived by validated functional outcome measures (short form 36 scores, Oswestry disability index) for patients who underwent discretionary surgery for specific degenerative conditions of the lumbar spine such as disc herniation, spinal stenosis, degenerative spondylolisthesis, and isthmic spondylolisthesis. After reviewing the records of all patients surgically treated for disc herniation, spinal stenosis, degenerative spondylolisthesis, and isthmic spondylolisthesis at our institution, we recorded the occurrence of PCSI (MRI or CT-myelograms) and reoperations, as well as demographic, surgical, and functional outcome data. We determined how early (within
\end{abstract}

D. Omoto - S. S. Bederman - A. J. M. Yee .

H. J. Kreder · J. A. Finkelstein

Division of Orthopaedic Surgery,

Sunnybrook Health Sciences Centre,

University of Toronto, Toronto, Canada

S. S. Bederman $(\bowtie)$

Department of Orthopaedic Surgery,

University of California at San Francisco,

500 Parnassus Avenue, MUW 3rd Floor,

San Francisco, CA 94143-0728, USA

e-mail: s.bederman@utoronto.ca
6 months) and intermediate (within 18 months) term events (PCSI and reoperations) were associated with changes in intermediate (minimum 1 year) and late (minimum 2 years) term functional outcome, respectively. We further evaluated how early (6-12 months) and intermediate (12-24 months) term changes in functional outcome were associated with the subsequent occurrence of intermediate (12-24 months) and late (beyond 24 months) term adverse events, respectively. From 148 surgically treated patients, we found no significant relationship between the occurrence of PCSI or reoperation and subsequent changes in functional outcome at intermediate or late term. Similarly, earlier changes in functional outcome did not have any significant relationship with subsequent occurrences of adverse events at intermediate or late term. Although it may be tempting to consider administrative database outcome measures as proxies for poor functional outcome, we cannot conclude that a significant relationship exists between the occurrence of PCSI or reoperation and changes in functional outcome.

Keywords Administrative data Postoperative imaging · Reoperation $\cdot$ Functional outcome $\cdot$ Spine surgery

\section{Introduction}

Administrative databases have been used increasingly to provide evidence for the effectiveness of surgical treatment for several degenerative conditions of the lumbar spine such as disc herniation, spinal stenosis, and degenerative spondylolisthesis [1, 6, 13-18, 23, 24]. Studies using administrative databases to investigate some of these conditions have provided us with knowledge about patterns and regional variations in utilization rates, characteristics of providers, and surgical outcomes [1, 6, 13-18, 23, 24]. 
These databases are a potential alternative to clinical trials because they are readily accessible, contain large numbers of patients, and allow for long-term follow-up [2, 21]. Despite the obvious appeal of administrative database research, there remain many problems. First, since most of these databases were designed for the provision of provider reimbursement and not for research purposes, as a result the quality of the data is not as good as prospectively collected clinical data $[2,7,10,12,22]$. Thus, conclusions drawn from low quality data can at best be of similar quality. Second, clinical significance may not necessarily follow from a large sample that yields statistical significance. However, with appropriate database validation and the analysis of clinically meaningful differences, these limitations are surmountable.

A significant limitation to the use of administrative databases, particularly as it relates to evaluating the effectiveness of lumbar spinal surgery, is the lack of patient-reported validated measures of functional outcome. Alternative outcomes such as reoperation rates, complications, hospital lengths of stay, and mortality may be interpreted by healthcare providers, researchers, or policymakers as surrogate measures or 'proxies' for poor functional outcome [1, 13, 15-18]. For the surgical treatment of these conditions, as in other discretionary procedures, these measures may not truly assess valid outcomes for patients [20].

Patients may undergo reoperation or secondary surgery either because of a clear complication from the index procedure or for more subjective indications based on the preferences and values of patients and surgeons. In some cases, surgeons may even plan 'staged' procedures whereby a second operation is contemplated based on the response to the first - a situation not necessarily consistent with a poor outcome from treatment $[5,19]$. In spinal surgery, it is quite common to 'stage' operations following a patient's response to a more conservative 'index' operation without necessarily experiencing clinical 'failure' [11].

Other outcome measures, apart from reoperations and complication rates, captured in administrative databases, not previously reported, may be better correlated, or may serve as a proxy, to patient-reported measures of functional outcome. Since most follow-up for spinal surgery, particularly for spinal fusion, includes simple radiographs to assess union, we hypothesized that advanced postoperative cross-sectional imaging (PCSI), such as magnetic resonance imaging (MRI) or computed tomography with myelography (CT-myelogram), would most commonly be indicated when patients have experienced significant postoperative complaints or complications (i.e., a poor clinical outcome).

The purpose of our study was to determine if an association between outcomes obtained from administrative databases, namely reoperations and PCSI, and validated measures of functional outcome exist for patients having undergone lumbar spinal surgery.

\section{Materials and methods}

Our clinical spinal program, at a tertiary care academic hospital, maintains a patient registry containing functional outcome measures for all spinal surgeries. Measures of functional outcome consists of modems short form 36 scores (SF-36) [22] and the Oswestry disability index (ODI) [8]. The study was performed following appropriate institutional research ethics approval. We identified all patients who underwent discectomy, decompression (i.e., laminectomy/laminotomy) or fusion for disc herniation, spinal stenosis, degenerative spondylolisthesis, or isthmic spondylolisthesis from 1 Jan 2000 to 31 Dec 2005 with complete measures of functional outcome. Patients who were treated for cervical or thoracic disease, tumors, infections, inflammatory disease, fractures, or major deformities requiring osteotomies were excluded from our analysis.

Medical records were reviewed and patient data were abstracted in a standardized fashion on patient demographics [age, gender, smoking status, and worker's compensation board status (WCB)], primary diagnosis, and surgical details. Patients were categorized according to the nature of their index procedure as either discectomy/ decompression or fusion (with or without decompression). We further abstracted data on postoperative events such as the need for and timing of PCSI and reoperations as well as pre and postoperative measures of functional outcome (SF-36, ODI).

From the SF-36, we calculated domains of bodily pain (BP) and physical function (PF) as well as physical component summary scores (PCS). We then determined the change in each domain from their baseline score at each time interval assessed to reflect patient-specific improvement. We conversely used the change in ODI score from baseline by subtracting the postoperative score from the preoperative baseline score to reflect their amount of improvement.

Data were analyzed using Student's $t$ tests and Chisquares for bivariate comparisons of continuous and categorical outcomes, respectively. Since functional outcome measures and adverse events (reoperations, PCSI) are timedependent variables, in order to determine their relationship we analyzed the data using two conceptual models. First, we analyzed how early (within 6 months) and intermediate term (within 18 months) events were associated with a subsequent change in intermediate (minimum 12 months) and late term (minimum 24 months) functional outcomes, 
respectively. For this, we used Student's $t$ tests for bivariate comparisons and multiple linear regression (since the dependent variables (SF-36, ODI) are continuous) controlling for patient demographics (age, gender, WCB, smoking status, and diagnosis) and surgical details (procedure and surgical extent). Second, we evaluated how early (6-12 months) and intermediate term (12-24 months) changes in functional outcome were associated with subsequent occurrence of intermediate (12-24 months) and late term (beyond 24 months) events, respectively, using logistic regression for the bivariate and multivariate models. Specifically, we used logistic regression to model the occurrence of either PCSI or reoperation as a binary variable explained by the change in functional outcome from baseline. Statistical analysis was performed using the SAS System v.9.1 (Cary, NC, USA).

\section{Results}

Baseline characteristics, adverse events and functional outcomes

We identified 148 patients that met the study criteria with pre and postoperative functional outcome measures (Fig. 1). Forty-five (30.4\%) patients underwent isolated discectomies, 51 (34.5\%) underwent spinal decompression, and $52(33.8 \%)$ underwent posterior lumbar fusion with or without adjunctive decompression. The distribution of patients requiring PCSI and reoperation is shown in Table 1.

We found no significant differences in age, gender, $\mathrm{WCB}$, smoking status, primary diagnosis, surgical procedure or extent between the group who received PCSI and those who did not. Similarly, we found no significant differences in demographics, diagnosis, or surgical details

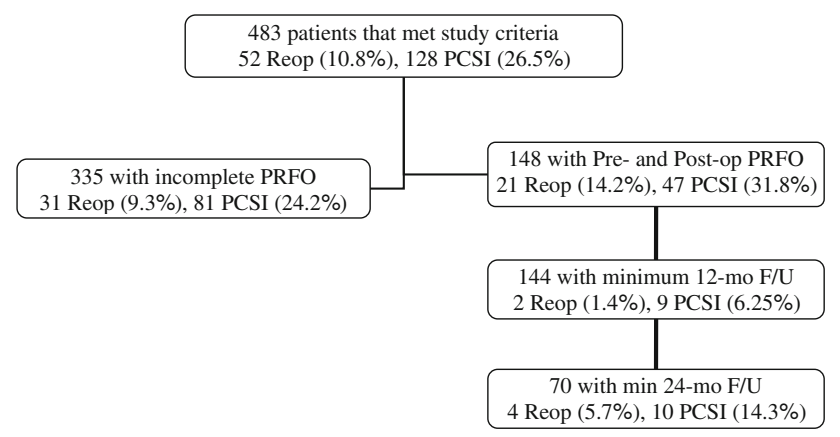

Reop - Reoperation

PCSI - Post-operative cross-sectional imaging

PRFO - Patient-Reported Functional Outcome

F/U - Follow-up

Fig. 1 Distribution of patients with occurrence of events between the patients who underwent reoperation compared with those that did not. Baseline scores of ODI were higher for the group that subsequently required PCSI compared with those who did not (41.8 vs. $27.5, P<0.005)$. Otherwise, there were no significant differences in baseline functional outcome scores for patients requiring subsequent imaging or reoperation (Table 1).

At a minimum of 1 and 2-year follow-up, we found significant improvements in all measures of functional outcome for the overall study cohort (Table 2). At 1-year follow-up, a greater improvement was identified in patients having single-level procedures compared with multi-level for all measures of outcome (BP $P<0.002$; ODI $P<0.01$; PCS $P<0.0004$; PF $P<0.004)$. A greater improvement in ODI was observed for patients who did not undergo fusion procedures compared with those that did $(P<0.02)$.

At 2-year follow-up, patients age 50 and under had greater improvement in PCS compared with those over 50 years of age $(P<0.05)$. Non-smokers had better improvements in PF $(P<0.04)$ and PCS $(P<0.04)$ at 2 years compared with smokers and patients who underwent single-level surgery had better improvement in BP compared with those who underwent multi-level procedures $(P<0.03)$. Otherwise, no other baseline patient characteristics were found to have a significant influence on functional improvement at the 1 and 2-year follow-up point.

Rates of PCSI and reoperations over the observation period are shown in Fig. 2. For both events, the distribution was left skewed such that the majority of events occurred within the first 12-24 months. Furthermore, the more frequent occurrence of PCSI peaked slightly earlier than the less frequent event of reoperation.

Multiple linear regression, controlling for all other predictors, demonstrated that the need for multilevel surgery was associated with a significant decline in BP and PCS at 1 year after surgery $(P<0.02)$, as shown in Table 3 . Younger age was also associated with a significant improvement in 1-year change in PCS, controlling for all other predictors $(P<0.03)$. Furthermore, patients who underwent spinal fusion were found to have a significantly lower change in ODI scores within 1 year compared with patients who underwent decompression/discectomy $(P<0.04)$.

Association between adverse events and changes in functional outcome

In comparing early PCSI and reoperation events to intermediate functional outcome change, we found no significant associations between these adverse events within 6 months and changes from baseline functional outcome at 1-year follow-up (Table 4). Similarly, PCSI and reoperation within 
Table 1 Baseline demographic, surgical and functional outcome data

\begin{tabular}{|c|c|c|c|c|c|}
\hline & \multirow[t]{2}{*}{ Total } & \multicolumn{2}{|l|}{ PCSI } & \multicolumn{2}{|l|}{ Reoperation } \\
\hline & & Yes & No & Yes & No \\
\hline Number $(\%)$ & 148 & $47(31.8)$ & $101(68.2)$ & $21(14.2)$ & $127(85.8)$ \\
\hline Mean age (SD) & $55.2(16.0)$ & $52.5(15.8)$ & $56.4(16.0)$ & $52.4(16.1)$ & $55.6(16.0)$ \\
\hline Female $(\%)$ & $79(53.4)$ & $28(18.9)$ & $51(75.0)$ & $12(8.1)$ & $67(45.3)$ \\
\hline WCB (\%) & $9(6.1)$ & $2(1.4)$ & $7(4.7)$ & $3(2.0)$ & $6(4.1)$ \\
\hline Smoking (\%) & $10(6.8)$ & $4(2.7)$ & $6(4.1)$ & $1(0.7)$ & $9(6.1)$ \\
\hline \multicolumn{6}{|l|}{ Primary diagnosis $(\%)$} \\
\hline Disc herniation & $57(38.5)$ & $21(44.7)$ & $36(35.6)$ & $9(42.9)$ & $48(37.8)$ \\
\hline Spinal stenosis & $46(31.1)$ & $14(29.8)$ & $32(31.7)$ & $5(23.8)$ & $41(32.3)$ \\
\hline Degenerative spondylolisthesis & $35(23.6)$ & $11(23.4)$ & $24(23.8)$ & $5(23.8)$ & $30(23.6)$ \\
\hline Isthmic spondylolisthesis & $10(6.8)$ & $1(2.1)$ & $9(8.9)$ & $2(9.5)$ & $8(6.3)$ \\
\hline Multilevel surgery (\%) & $59(39.9)$ & $18(12.2)$ & $41(27.7)$ & $11(7.4)$ & $48(32.4)$ \\
\hline \multicolumn{6}{|l|}{ Procedure $(\%)$} \\
\hline Discectomy & $45(30.4)$ & $17(36.2)$ & $28(27.7)$ & $8(38.1)$ & $37(29.1)$ \\
\hline Decompression & $51(34.5)$ & $17(36.2)$ & $34(33.7)$ & $7(33.3)$ & $44(34.7)$ \\
\hline Fusion & $52(35.1)$ & $13(27.7)$ & 39 (38.6) & $6(28.6)$ & $46(36.2)$ \\
\hline \multicolumn{6}{|l|}{ Baseline functional outcome (SD) } \\
\hline $\mathrm{BP}$ & $21.9(16.0)$ & $19.8(15.1)$ & $23.0(16.3)$ & $21.0(17.9)$ & $22.1(15.7)$ \\
\hline $\mathrm{PF}$ & $28.8(22.9)$ & $27.2(22.0)$ & $28.7(22.5)$ & $25.0(19.9)$ & $28.8(22.7)$ \\
\hline PCS & $25.4(10.3)$ & $24.2(9.6)$ & $26.2(10.7)$ & $24.1(8.5)$ & $25.8(10.7)$ \\
\hline ODI & $21.2(27.6)$ & $41.8(27.6)^{*}$ & $27.5(27.1)^{*}$ & $38.3(24.3)$ & $30.9(28.5)$ \\
\hline
\end{tabular}

Null hypothesis: no difference between groups (PCSI-Yes vs. PCSI-No and Reoperation-Yes vs. Reoperation-No) for baseline data

PCSI Postoperative cross-sectional imaging, SD standard deviation, WCB worker's compensation board claim, $B P$ mean change in the bodily pain index (SF-36), PF mean change in the physical function index (SF-36), PCS mean change in physical component summary score (SF-36), $O D I$ mean change in Oswestry disability index

* Significant at the $P<0.005$ level

18 months after surgery were not associated with functional outcome change beyond 2-year follow-up (Table 4).

In comparing early changes in functional outcome (i.e., 6-12 months after surgery) with the occurrence of PCSI or reoperation at 12-24 months postoperatively, we found no significant statistical associations (Table 5). Similarly, change in functional outcome during the second postoperative year (i.e., 12-24 months) was also not statistically associated with the occurrence of PCSI or reoperation beyond 2 years after the index operation (Table 5).

Post hoc power calculations were performed for the detection of differences in functional outcome change. For 21 patients who underwent reoperation (and 47 who underwent PCSI) and using standardized response means (=mean change/SD) of 0.7 and 0.84 for SF-36 PCS and ODI, respectively, the power to detect a difference in functional outcome between an equivalent size group is $99 \%$ [4]. In the case in which early events were compared with later functional outcome changes, the number of events were small, particularly for reoperations ( $n=2$ for within 6 months and $n=4$ within 18 months). Obviously, these numbers would be well underpowered to detect a clinically meaningful difference in clinical outcomes. However, at the 24-month time period, the point estimate of the mean change in functional outcome was higher for those who underwent reoperation (BP 27.5, physical function 28.8, and PCS 13.4) compared with those who did not (SF-36 BP 24.6, physical function 20.9, and PCS 9.3) suggesting that even with more precision, the relationship is unlikely to be significant in a negative direction. For a sample size of 9 (PCSI within 6 months), and a standardized response mean of 0.7 and 0.84 the power is 79 and $91 \%$ that a significant difference will be detected for SF-36 PCS and ODI at a minimum of 12 months, respectively, and at the minimum 24-month time point (PCSI, $n=10$ within 18 months) the power would be even higher [4].

\section{Discussion}

Surgery, for many conditions affecting the lumbar spine, including disc herniation, spinal stenosis, and degenerative spondylolisthesis, is beneficial in selected patients [25, 27, 28]. Large administrative database research has 


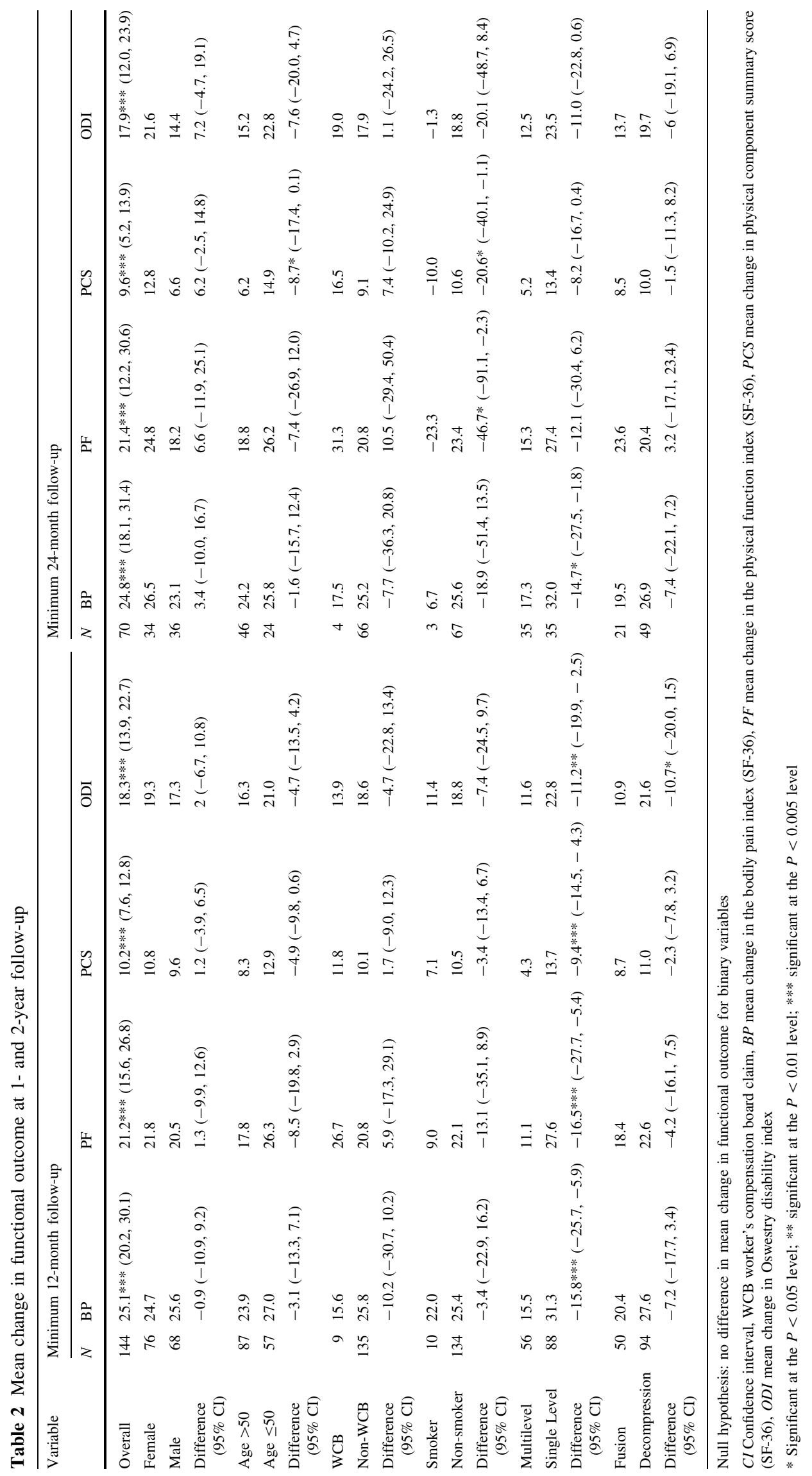


Fig. 2 Frequency of repeat imaging and reoperations over time

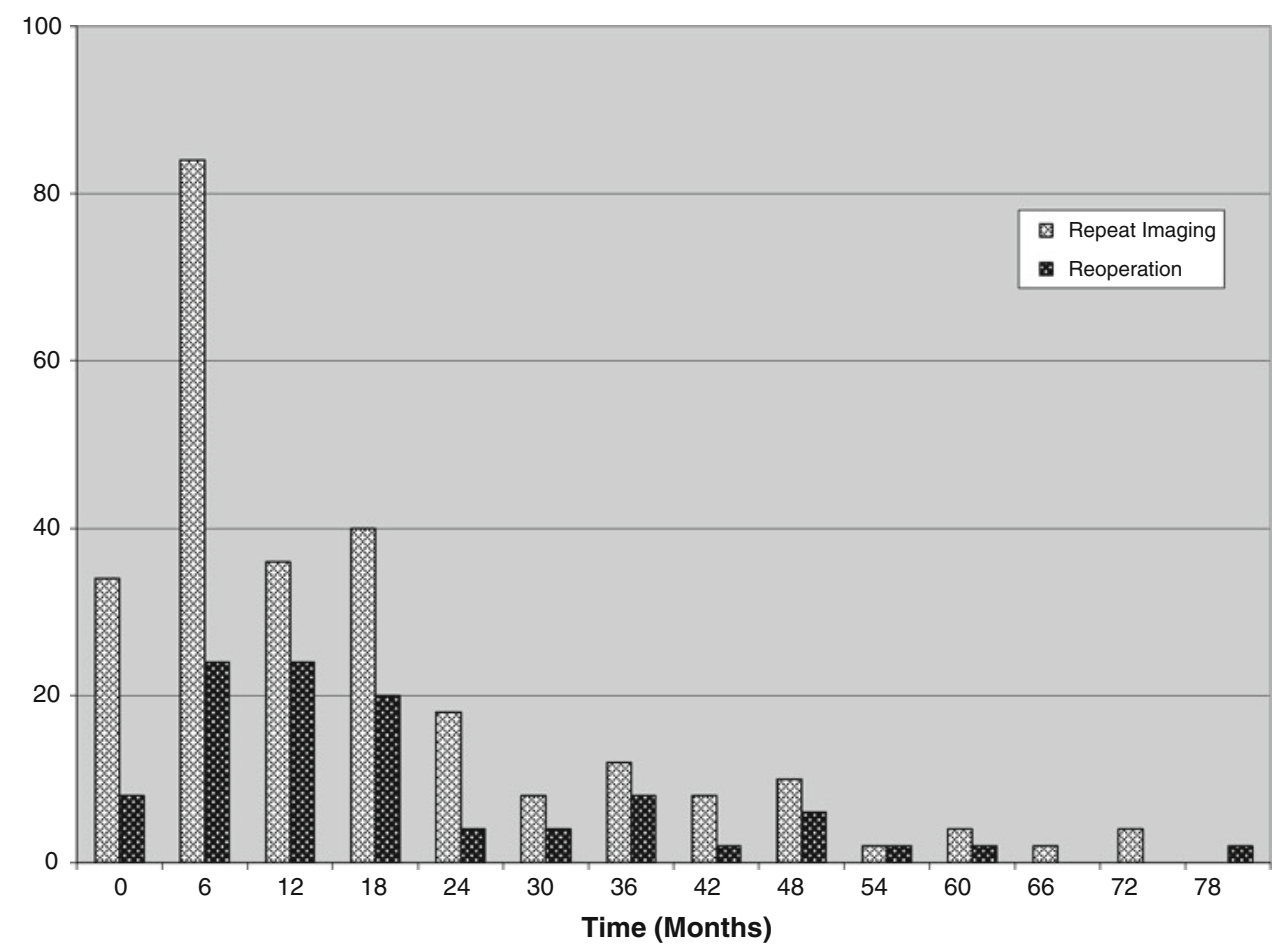

Table 3 Results from multiple linear regression of baseline variables on change in functional outcome at minimum 1 year

\begin{tabular}{llll}
\hline Functional outcome & Covariate & Estimate $(95 \%$ confidence interval) & P value \\
\hline BP & Multilevel disease & $-13.6(-16.5,-10.8)$ & 0.018 \\
PCS & Age at surgery (years) & $-0.2(-0.25,-0.15)$ & 0.027 \\
& Multilevel disease & $-6.9(-8.3,-5.5)$ & 0.015 \\
ODI & Fusion & $-10.1(-12.5,-7.7)$ & 0.039
\end{tabular}

$B P$ change in the bodily pain index from the SF-36, ODI Oswestry disability index change, $P C S$ physical component summary score change of the SF-36

helped us understand patterns of utilization of health services for these conditions [1, 6, 13-18, 23, 24].

Keskimaki et al. [14] found that there was substantial regional variation in lumbar disc surgery rates across Finnish hospital regions. The same authors later looked at reoperation rates following disc surgery in Finland and found a 9-year reoperation rate of $18.9 \%$ [15]. Furthermore, the authors found that reoperation rates were slightly higher for neurosurgeons compared with orthopedic surgeons. Hu et al. [13] used administrative databases to determine population-based estimates of reoperation rates in a Canadian province. The authors found that the overall reoperation rate was $9.5 \%$ in their cohort of 4,722 patients and that it was slightly higher in patients who underwent fusion compared with decompression alone. Bederman et al. [1] further examined reoperation rates in the same region and found a reoperation rate of $10.6 \%$. In their analysis, they found a slightly higher reoperation rate for fusions over decompression at 2 years but not in the longterm. Long-term survival analysis demonstrated that lower surgical volume had a higher reoperation rate while surgeon specialty was not found to have any significant relationship. Malter et al. [16] looked at reoperation rates in the State of Washington over a 5-year period. The authors found that overall, fusions had a higher rate of reoperation (18\%) compared with decompressions (15\%), but this finding was not significant in the multivariable regression model.

Overall, there were significant improvements in all measures of functional outcome in our study cohort up to 2 years (BP 24.8; PF 21.4; PCS 9.6; ODI 17.9). Carreon et al. [3] in their systematic review of patients with degenerative lumbar disease found similar improvements in ODI (18.3) and SF-36 PCS (9.4) in surgical patients. Their review of a predominantly back-dominant pain cohort included diagnoses of degenerative disc disease, chronic low back pain, and spondylolisthesis. In the recent spine patient outcomes research trials (SPORT) for disc herniation, degenerative spondylolisthesis, and spinal stenosis, the authors found that the as-treated surgical groups 
Table 4 Linear regression results of occurrence of adverse events (PCSI, reoperation) on subsequent change in functional outcome

\begin{tabular}{|c|c|c|c|c|c|}
\hline & $N$ & $\mathrm{BP}$ & $\mathrm{PF}$ & PCS & ODI \\
\hline \multicolumn{6}{|l|}{ Early event } \\
\hline \multicolumn{6}{|c|}{ Minimum 12-month functional outcome by events within 6 months } \\
\hline PCSI (SD) & 9 & $22.2(33.1)$ & $6.7(32.5)$ & $9.7(11.6)$ & $6.3(21.5)$ \\
\hline No PCSI (SD) & 135 & $25.3(29.9)$ & $22.1(34.0)$ & $10.3(14.9)$ & $19.0(25.0)$ \\
\hline Difference $(95 \% \mathrm{CI})$ & & $-3.1(-23.7,17.4)$ & $-15.4(-38.5,7.6)$ & $-0.6(-11.2,10.1)$ & $-12.7(-31.9,6.5)$ \\
\hline Reoperation (SD) & 2 & $10.0(14.1)$ & $12.5(53.0)$ & $16(-)$ & $36.0(-)$ \\
\hline No reoperation $(\mathrm{SD})$ & 142 & $25.4(30.2)$ & $21.3(33.9)$ & $10.2(14.7)$ & $18.2(25.0)$ \\
\hline Difference $(95 \% \mathrm{CI})$ & & $-15.4(-57.8,27.1)$ & $-8.8(-56.8,39.2)$ & $5.8(-23.4,35.1)$ & $17.8(-31.9,67.6)$ \\
\hline \multicolumn{6}{|l|}{ Intermediate event } \\
\hline \multicolumn{6}{|c|}{ Minimum 24-month functional outcome by events within 18 months } \\
\hline PCSI (SD) & 10 & $15.0(17.2)$ & $11.0(40.7)$ & $6.3(15.8)$ & $9.5(20.2)$ \\
\hline No PCSI (SD) & 59 & $26.4(28.8)$ & $23.1(38.3)$ & $10.1(17.2)$ & $19.4(24.9)$ \\
\hline Difference $(95 \% \mathrm{CI})$ & & $-11.4(-30.2,7.4)$ & $-12.1(-38.5,14.3)$ & $-3.8(-16.2,8.4)$ & $-9.9(-26.6,6.8)$ \\
\hline Reoperation (SD) & 4 & $27.5(15.0)$ & $28.8(53.6)$ & $13.4(14.6)$ & $17.3(22.6)$ \\
\hline No reoperation (SD) & 65 & $24.6(28.3)$ & $20.9(38.0)$ & $9.3(17.1)$ & $18.0(24.7)$ \\
\hline Difference $(95 \% \mathrm{CI})$ & & $2.9(-25.7,31.5)$ & $7.9(-32.1,47.8)$ & $4.1(-13.5,21.7)$ & $-0.7(-26.0,24.6)$ \\
\hline
\end{tabular}

Null hypothesis: no difference in mean change in functional outcome for events (PCSI, reoperation)

$S D$ Standard deviation, $C I$ confidence Interval, $B P$ mean change in the bodily pain index (SF-36), $P F$ mean change in the physical function index (SF-36), PCS mean change in physical component summary score (SF-36), ODI mean change in Oswestry disability index, PCSI postoperative cross-sectional imaging

Table 5 Logistic regression results of change in functional outcome on subsequent occurrence of adverse events (PCSI, reoperation)

\begin{tabular}{lcr}
\hline Functional outcome & $\begin{array}{l}\text { PCSI odds ratio } \\
\text { (95\% confidence intervals) }\end{array}$ & $\begin{array}{l}\text { Reoperation odds } \\
\text { (95\% confidence inte }\end{array}$ \\
\hline Occurrence of events within 12-24 months by functional outcome change at 6-12 months \\
BP & $0.99(0.97,1.01)$ & $1.02(0.99,1.05)$ \\
PF & $0.99(0.98,1.01)$ & $1.03(1.00,1.07)$ \\
PCS & $0.98(0.93,1.04)$ & $1.02(0.93,1.11)$ \\
ODI & $1.01(0.98,1.03)$ & $1.03(0.98,1.08)$ \\
Occurrence of events beyond 24 months by functional outcome change at $12-24$ months & $1.02(0.98,1.05)$ \\
BP & $1.00(0.97,1.02)$ & $0.99(0.96,1.02)$ \\
PF & $0.99(0.96,1.01)$ & $1.02(0.95,1.09)$ \\
PCS & $0.98(0.93,1.03)$ & $1.00(0.97,1.04)$ \\
ODI & $0.99(0.95,1.02)$ &
\end{tabular}

Odds ratios represent the incremental change in odds of adverse event relative to no event for each unit change in functional outcome score $P C S I$ Postoperative cross-sectional imaging, $B P$ mean change in the bodily pain index (SF-36), $P F$ mean change in the physical function index (SF-36), PCS mean change in physical component summary score (SF-36), ODI mean change in Oswestry disability index

improved significantly by 2 years [25-27]. Improvements for SF-36 BP was 42.6, 29.9, and 26.9, for physical function was 43.9, 26.6, and 23.0, and for ODI was 24.2, 24.2, and 20.5 , respectively, for disc herniation, degenerative spondylolisthesis, and spinal stenosis.

In the current study, the overall reoperation rate was $14.2 \%$ and the rate of PCSI was $31.8 \%$. Fritzell et al. [9] reported on the results of a multicentred study from the Swedish Lumbar Spine Study Group that included 211 patients with chronic low back pain treated with either posterolateral non-instrumented, instrumented, or interbody fusion. The authors found a $14.7 \%$ reoperation rate while their systematic review demonstrated a wide range of reoperation rates $(0-40 \%)$. Furthermore, they found no statistical associations between the occurrence of complications and differences in functional outcome.

Based on this analysis, we cannot conclude that there is any association between the occurrence of certain adverse 
events, such as reoperation or PCSI, and change in functional outcome. Although the relationship between these time-dependent measures of outcome can be complex, we found no significant associations with functional outcome change and the subsequent occurrence of adverse events or, conversely, with the occurrence of adverse events and the association of subsequent changes in functional outcome. We should, therefore, temper our interpretation of results obtained from administrative data when assessing meaningful outcomes as they relate to patient function $[1,13$, $15-18]$.

The main limitation of this study, similar to other observational studies, is that the initial collection of functional outcome data was not complete for all patients surgically treated and thus may have been prone to selection bias. Some patients may have been followed on an "as needed basis" early in their recovery period, presumably due to accelerated progress, and may not have been captured in the functional outcome database or patients who may have sought care elsewhere for poor recovery were also not captured in our database. Since our facility is a major referral centre, many patients from remote areas who underwent surgery did not return for follow-up. The net effect this may have on our overall results is not entirely clear. Nonetheless, in comparing the patient demographics of those with functional outcome measures and those without, we found no differences in rates of reoperation or PCSI, suggesting that the group with complete outcome data is generally representative of the entire group and not significantly biased by the exclusion of those lost to follow-up. Furthermore, the rate of reoperation $(14.2 \%)$ found in this series was consistent with other studies based on administrative databases (9.5-19\%), further suggesting that our study population was representative of a typical patient population undergoing elective lumbar spinal surgery $[1,13,15-18]$.

Another limitation is the small number of patients who underwent reoperation or required PCSI. Overall, this study was well powered to detect a difference in functional outcome measures. The number of events was small for reoperations and therefore underpowered to detect a clinically meaningful difference in clinical outcomes, however, the relationship suggested an improved functional outcome for those patients who underwent reoperation further supporting our findings.

One final limitation is that our results may not necessarily generalize to other regions particularly for the case of PCSI. Although the decision to undergo reoperation is clearly discretional based on the values of patients and surgeons, the general indications are relatively similar across regions as demonstrated by similar reoperation rates in other studies $[1,13,15,16]$. Furthermore, reoperation rates at our institution are not significantly different from other hospitals across the province of Ontario. However, no reports on the use of PCSI have been published. The use of PCSI is not only discretional based on similar values of patients and their surgeons but also dependent on the health care system in which one practices. Financial disincentives, cost containment measures, and access to imaging scanners pose additional barriers to their use suggesting that there may be more variation in the use of PCSI among different countries than in the need for reoperations. Nonetheless, in Canada, physicians practice in a fee-for-service system whereby surgeons may order tests entirely at their discretion. But since the system is publicly funded, there exists a responsibility on physicians to constrain overall costs incurred, suggesting that our findings may lie in a central location within the spectrum of global healthcare systems.

In summary, we cannot conclude that a significant relationship exists between validated patient-derived measures of functional outcome after lumbar spinal surgery and the need for PCSI or reoperations. Although it is tempting for researchers to be able to use these measures obtainable in administrative data as proxy measures for functional outcome, we cannot conclude that a clear relationship exists. Other associations between outcomes from administrative databases and patient-reported measures of outcomes warrant further study.

\section{Conclusions}

Although it may be tempting to consider administrative database outcome measures as proxies for functional outcome, we cannot conclude that a significant relationship exists between the occurrence of PCSI or reoperation and changes in functional outcome.

Acknowledgments Bederman S.S. was supported by the OREF/ AAOS Clinical Research Training Fellowship.

Open Access This article is distributed under the terms of the Creative Commons Attribution Noncommercial License which permits any noncommercial use, distribution, and reproduction in any medium, provided the original author(s) and source are credited.

\section{References}

1. Bederman SS, Kreder HJ, Weller I, Finkelstein JA, Ford M, Yee AJM (2009) The who, what, and when of surgery for the degenerative lumbar spine. A population-based study of surgeon factors, surgical procedures, recent trends and reoperation rates. Can J Surg 52(4):283-290

2. Billings J (2003) Using administrative data to monitor access, identify disparities and assess performance of the safety net. Tools for monitoring the health care safety net. Agency for Healthcare Research and Quality http://www.ahrq.gov/data/ safetynetbillings.htm 
3. Carreon LY, Glassman SD, Howard J (2008) Fusion and nonsurgical treatment for symptomatic lumbar degenerative disease: a systematic review of Oswestry Disability Index and MOS Short Form-36 outcomes. Spine J 8:747-755

4. Copay AG, Glassman SD, Subach BR, Berven S, Schuler TC, Carreon LY (2008) Minimum clinically important difference in lumbar spine surgery patients: a choice of methods using the Oswestry Disability Index, Medical Outcomes Study questionnaire Short Form 36, and Pain Scales. Spine J 8:968-974

5. Dewald CJ, Millikan KW, Hammerberg KW, Doolas A, Dewald RL (1999) An open, minimally invasive approach to the lumbar spine. Am Surg 65:61-68

6. Deyo RA, Gray DT, Kreuter W, Mirza S, Martin BI (2005) United States trends in lumbar fusion surgery for degenerative conditions. Spine 30:1441-1445

7. Dismuke CE (2005) Underreporting of computed tomography and magnetic resonance imaging procedures in inpatient claims data. Med Care 43:713-717

8. Fairbank JC, Couper J, Davies JB, O'Brien JP (1980) The Oswestry low back pain disability questionnaire. Physiotherapy 66:271-273

9. Fritzell P, Hagg O, Nordwall A (2003) Complications in lumbar fusion surgery for chronic low back pain: comparison of three surgical techniques used in a prospective randomized study. A report from the Swedish Lumbar Spine Study Group. Eur Spine J 12:178-189

10. Fulton-Kehoe D, Gluck J, Wu R, Mootz R, Wickizer TM, Franklin GM (2007) Measuring work disability: what can administrative data tell us about patient outcomes? J Occup Environ Med 49:651-658

11. Hansraj KK, O'Leary PF, Cammisa FP Jr, Hall JC, Fras CI, Cohen MS, Dorey FJ (2001) Decompression, fusion, and instrumentation surgery for complex lumbar spinal stenosis. Clin Orthop Relat Res 384:18-25

12. Henderson T, Shepheard J, Sundararajan V (2006) Quality of diagnosis and procedure coding in ICD-10 administrative data. Med Care 44:1011-1019

13. Hu RW, Jaglal S, Axcell T, Anderson G (1997) A populationbased study of reoperations after back surgery. Spine 22:22652271

14. Keskimaki I, Aro S, Teperi J (1994) Regional variation in surgical procedure rates in Finland. Scand J Soc Med 22:132-138

15. Keskimaki I, Seitsalo S, Osterman H, Rissanen P (2000) Reoperations after lumbar disc surgery: a population-based study of regional and interspecialty variations. Spine 25:1500-1508

16. Malter AD, McNeney B, Loeser JD, Deyo RA (1998) 5-year reoperation rates after different types of lumbar spine surgery. Spine 23:814-820
17. Martin BI, Mirza SK, Comstock BA, Gray DT, Kreuter W, Deyo RA (2007) Are lumbar spine reoperation rates falling with greater use of fusion surgery and new surgical technology? Spine 32:2119-2126

18. Martin BI, Mirza SK, Comstock BA, Gray DT, Kreuter W, Deyo RA (2007) Reoperation rates following lumbar spine surgery and the influence of spinal fusion procedures. Spine 32:382-387

19. McPhee IB, Swanson CE (1998) The surgical management of degenerative lumbar scoliosis. Posterior instrumentation alone versus two stage surgery. Bull Hosp Jt Dis 57:16-22

20. Pollock H, Pollock T (2007) Is reoperation rate a valid statistic in cosmetic surgery? Plast Reconstr Surg 120:569

21. Romano PS, Schembri ME, Rainwater JA (2002) Can administrative data be used to ascertain clinically significant postoperative complications? Am J Med Qual 17:145-154

22. Ware JE Jr, Sherbourne CD (1992) The MOS 36-item short-form health survey (SF-36). I. Conceptual framework and item selection. Med Care 30:473-483

23. Weinstein JN, Bronner KK, Morgan TS, Wennberg JE (2004) Trends and geographic variations in major surgery for degenerative diseases of the hip, knee, and spine. Health Aff (Millwood) Supplemental Web Exclusives:VAR81-VAR89

24. Weinstein JN, Lurie JD, Olson PR, Bronner KK, Fisher ES (2006) United States' trends and regional variations in lumbar spine surgery: 1992-2003. Spine 31:2707-2714

25. Weinstein JN, Lurie JD, Tosteson TD, Hanscom B, Tosteson AN, Blood EA, Birkmeyer NJ, Hilibrand AS, Herkowitz H, Cammisa FP, Albert TJ, Emery SE, Lenke LG, Abdu WA, Longley M, Errico TJ, Hu SS (2007) Surgical versus nonsurgical treatment for lumbar degenerative spondylolisthesis. N Engl J Med 356:22572270

26. Weinstein JN, Lurie JD, Tosteson TD, Skinner JS, Hanscom B, Tosteson AN, Herkowitz H, Fischgrund J, Cammisa FP, Albert T, Deyo RA (2006) Surgical vs nonoperative treatment for lumbar disk herniation: the Spine Patient Outcomes Research Trial (SPORT) observational cohort. JAMA 296:2451-2459

27. Weinstein JN, Tosteson TD, Lurie JD, Tosteson AN, Blood E, Hanscom B, Herkowitz H, Cammisa F, Albert T, Boden SD, Hilibrand A, Goldberg H, Berven S, An H (2008) Surgical versus nonsurgical therapy for lumbar spinal stenosis. N Engl J Med 358:794-810

28. Weinstein JN, Tosteson TD, Lurie JD, Tosteson AN, Hanscom B, Skinner JS, Abdu WA, Hilibrand AS, Boden SD, Deyo RA (2006) Surgical vs nonoperative treatment for lumbar disk herniation: the Spine Patient Outcomes Research Trial (SPORT): a randomized trial. JAMA 296:2441-2450 